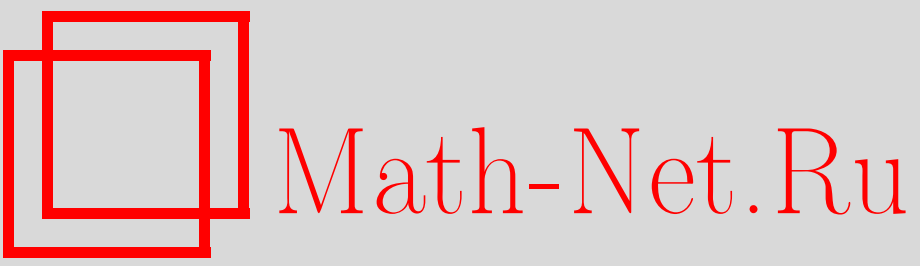

Г. А. Сарданашвили, Классическая калибровочная теория гравитации, ТМФ, 2002, том 132, номер 2, 318-328

DOI: https://doi.org/10.4213/tmf364

Использование Общероссийского математического портала Math-Net.Ru подразумевает, что вы прочитали и согласны с пользовательским соглашением

http://www . mathnet.ru/rus/agreement

Параметры загрузки:

IP : 54.162 .27 .143

26 апреля 2023 г., $14: 24: 32$ 
ТЕОРЕТИЧЕСКАЯ

И МАТЕМАТИЧЕСКАЯ

ФИЗИКА

Том 132, № 2

август, 2002

(C) 2002 г.

Г. А. Сарданашвили*

\section{КЛАССИЧЕСКАЯ КАЛИБРОВОЧНАЯ ТЕОРИЯ ГРАВИТАЦИИ}

Классическая теория гравитации формулируется как калибровочная теория на реперном расслоении со спонтанным нарушением симметрий, обусловленным существованием дираковских фермионных полей. Соответствующим хиггсовским полем является псевдориманова метрика (тетрадное поле). Рассматриваются два варианта этой теории. В первом варианте гравитация представляется псевдоримановой метрикой, как в общей теории относительности, во втором - эффективной метрикой, как в релятивистской теории гравитации А. А. Логунова. Для обоих вариантов строятся конфигурационное пространство, оператор Дирака и лагранжианы.

Ключевые слова: гравитация, калибровочное поле, хиггсовское поле, спинорное поле.

\section{1. ВВЕДЕНИЕ}

Первая калибровочная модель гравитации [1] появилась спустя всего два года после рождения самой калибровочной теории. Однако первоначальные попытки построения калибровочной теории гравитации по аналогии с калибровочными моделями внутренних симметрий столкнулись с серьезными трудностями - установлением калибровочного статуса метрического (тетрадного) поля и выбором калибровочной группы. В работе [1] гравитация описывалась в рамках калибровочной модели группы Лоренца, но тетрадные поля вводились произвольно. Чтобы устранить этот недостаток, тетрадные поля попытались представить как калибровочные поля группы трансляций [2]-[9], но безуспешно. Сначала в качестве генераторов калибровочной группы трансляций рассматривался канонический лифт векторных полей на многообразии $X$ на касательное расслоение $T X[2]$, [3], который на самом деле является генератором обших ковариантных преобразований $T X$. Затем в таком же качестве предлагался горизонтальный лиф̆т векторных полей на $X$ посредством линейной связности на $T X \rightarrow X[4]$. Позже тетрадное поле стали отождествлять с трансляционной частью аффинной связности на касательном расслоении $T X \rightarrow X$. Всякая такая связность представляет собой сумму линейной связности и тангенциальнозначной формы

$$
\Omega=\Omega_{\lambda}^{\alpha}(x) d x^{\lambda} \otimes \partial_{\alpha}
$$

\footnotetext{
* Московский государственный университет, Москва, Россия. E-mail: sard@grav.phys.msu.su
} 
но эта форма и тетрадное поле являются разными математическими объектами [10], [11] . Заметим, что форма (1) продолжает фигурировать в одном из вариантов калибровочной теории гравитации как неголономный репер [12]. Вместе с тем оказалось, что трансляционные связности описывают дисторсию в калибровочной теории дислокаций [13], [14] и в аналогичной калибровочной модели “пятой силы" [11], [15].

Трудности в построении калибровочной теории гравитации по аналогии с калибровочной теорией внутренних симметрий были обусловлены тем, что калибровочные преобразования в этих теориях принадлежат разным классам. В случае внутренних симметрий калибровочные преобразования - это вертикальные автоморфизмы главного расслоения $P \rightarrow X$, оставляющие неподвижной его базу $X$. Теория же гравитации строится на главном расслоении $L X$ касательных реперов к $X$ и ассоциированных с ним тензорных расслоениях к $X$. Они принадлежат категории расслоений $T \rightarrow X$, для которых диффееморфизмы $f$ базы $X$ канонически порождают автоморфизмы $\tilde{f}$ расслоения $T$. Эти автоморфизмы называются голономными автоморфизмами или общими ковариантными преобразованиями. Все гравитационные лагранжианы конструируются инвариантными относительно обших ковариантных преобразований, которые тем самым являются калибровочными преобразованиями теории гравитации. Можно рассмотреть и неголономные (например, вертикальные) автоморфизмы главного реперного расслоения $L X$, но большинство гравитационных лагранжианов не остаются инвариантными при этих преобразованиях.

В настойчивых попытках представить тетрадноеполе как калибровочное упускалось из виду, что в калибровочной теории со спонтанным нарушением симметрий, помимо материальных и калибровочных полей, присутствуют еше хиггсовские поля. Спонтанное нарушение симметрий - квантовый эффект, при котором вакуум не инвариантен относительно группы преобразований. В терминах классической калибровочной теории спонтанное нарушение симметрий имеет место, если структурная группа $G$ главного расслоения $P \rightarrow X$ редуцируема к замкнутой подгруппе $H$, т.е. сушествует главное подрасслоение расслоения $P$ со структурной группой $H$ [10], [16]-[18]. Такая редукция имеет место тогда и только тогда, когда фактор-расслоение $P / H \rightarrow X$ допускает глобальное сечение. Более того, существует взаимно однозначное соответствие между редуцированными подрасслоениями $P^{h} \subset P$ со структурной группой $H$ и глобальными сечениями $h$ фактор-расслоения $P / H \rightarrow X$ [19]. Последние трактуются как классические хиггсовские поля.

Представление о псевдоримановой метрике как хиггс-голдстоуновском поле зародилось при построении нелинейных (индуцированных) представлений группы $G L(4, R)$, картановской подгруппой которой является группа Лоренца [20], [21]. Когда калибровочная теория была сформулирована в терминах расслоений, само определение псевдоримановой метрики на многообразии $X$ как глобального сечения фактор-расслоения

$$
\Sigma_{P R}=L X / S O(1,3) \rightarrow X
$$

подвело к ее физической интерпретации как хиггсовского поля, ответственного за спонтанное нарушение пространственно-временных симметрий [10], [22], [23]. Теоретичес- 
ким обоснованием такого нарушения симметрий служит геометрический принцип эквивалентности, постулирующий сушествование системы отсчета, в которой лоренцевские инварианты определены на всем пространственно-временном многообразии [10]. В терминах расслоений это означает, что структурная группа $G L(4, R)$ реперного и ассоциированных с ним расслоений редуцирована к группе Лоренца.

Физической причиной нарушения пространственно-временных симметрий является сушествование дираковской фермионной материи с группой симметрий - универсальным двулистным накрытием $L_{s}=S L(2, C)$ собственной группы Лоренца $L=S O^{\uparrow}(1,3)$. Дираковская спиновая структура на пространственно-временном многообразии $X$ определяется как пара $\left(P^{h}, z_{\mathrm{s}}\right)$ главного расслоения $P^{h} \rightarrow X$ со структурной группой $L_{s}$ и его послойного морфизма

$$
z_{s}: P^{h} \rightarrow L X
$$

в реперное расслоение $L X$ [24], [25]. Всякий такой морфизм факторизуется посредством морфизма

$$
z_{h}: P^{h} \rightarrow L^{h} X
$$

где $L^{h} X$ - некоторое главное подрасслоение реперного расслоения $L X$ со структурной группой Лоренца $L$.

Предположим в дальнейшем, что $X-4$-мерное ориентированное (односвязное, гладкое, отделимое, локально-компактное счетное в бесконечности, т.е. паракомпактное) многообразие с координатным атласом $\left\{\left(U_{\zeta}, x^{\lambda}\right)\right\}$ и что $L X$ - расслоение ориентированных касательных реперов к $X$ со структурной группой $G L_{4}=G L^{+}(4, R)$. Тогда имеет место взаимно однозначное соответствие между упомянутыми выше редуцированными расслоениями $L^{h} X$ со структурной группой $L$ и глобальными сечениями $h$ фактор-расслоения

$$
\Sigma=L X / L \rightarrow X
$$

с типичным слоем $G L_{4} / L$. Иными словами, сужение расслоения $L X \rightarrow \Sigma$ на $h(X) \subset \Sigma$ изоморфно $L^{h} X$. Расслоение (5) является двулистным накрытием расслоения $\Sigma_{P R}(2)$. Его глобальное сечение $h$ называется тетрадным полем. Оно представляется набором локальных сечений $h_{\mu}^{a}(x)$ редуцированного подрасслоения $L^{h} X$, именуемых тетрадными функциями. Всякое тетрадное поле однозначно определяет псевдориманову метрику на $X$ так, что выполняется известное соотношение

$$
g_{\mu \nu}=h_{\mu}^{a} h_{\nu}^{b} \eta_{a b}
$$

где $\eta$ - метрика Минковского. Таким образом, дираковская спиновая структура на пространственно-временном многообразии однозначно задает на нем псевдориманову метрику.

Существование дираковской спиновой структуры накладывает также жесткие ограничения на топологию пространственно-временного многообразия $X[26],[27]$. Поскольку компактное пространство-время не удовлетворяет какому-либо принципу причинности, предположим в дальнейшем, что многообразие $X$ некомпактно. Тогда оно параллелизуемо, т.е. реперное расслоение $L X \rightarrow X$ тривиально. В этом случае все спиновые 
структуры $P^{h}$ на $X$ взаимно изоморфны, хотя этот изоморфизм не является каноническим.

Обозначим через $S^{h} \rightarrow X$ спинорное расслоение, ассоциированное с главным расслоением $P^{h}$. Его сечения описывают дираковские фермионные поля в присутствии тетрадного поля $h$. Проблема состоит в том, что фермионные поля в присутствии разных тетрадных полей представляются сечениями разных расслоений, между которыми нет канонического изоморфизма. Более того, чтобы определить оператор Дирака на сечениях расслоения $S^{h}$, необходимо задать представление

$$
d x^{\lambda} \mapsto \gamma_{h}\left(d x^{\lambda}\right)=h_{a}^{\lambda}(x) \gamma^{a}
$$

касательных кореперов $\left\{d x^{\lambda}\right\}$ к пространственно-временному многообразию $X$ матрицами Дирака. Однако для разных тетрадных полей $h$ эти представления не эквивалентны. Именно в этом проявляется хиггсовский характер классического тетрадного поля. Таким образом, дираковское фермионное поле на пространственно-временном многообразии может рассматриваться только в паре с определенным тетрадным полем.

В частности, поскольку морфизм (3) факторизуется посредством морфизма (4), вся сушествуюшая фермионная материя, представляемая сечениями некоторого спинорного расслоения над $X$, однозначно фиксирует лоренцевскую структуру $L^{h} X$ и тетрадное поле $h$ (псевдориманову метрику $g$ ) на $X$, причем независимо от динамики фермионных полей. Поэтому это тетрадное поле (псевдориманова метрика) не определяется какими-либо дифференциальными уравнениями, а является фоновым. Следовательно, оно не может отождествляться с гравитационным полем и в теории гравитации присутствует фоновая геометрия. Эти соображения побуждают рассмотреть два варианта калибровочной теории гравитации.

В первом варианте [11], [28]-[31] гравитационное поле отождествляется с тетрадным полем и рассматривается композиционное расслоение

$$
S \rightarrow \Sigma \rightarrow X
$$

где $S \rightarrow \Sigma$ - спинорное расслоение, ассоциированное с главным расслоением $L X \rightarrow \Sigma$ со структурной группой Лоренца $L$. Идея данного построения состоит в том, что для всякого сечения $h$ тетрадного расслоения $\Sigma \rightarrow X$ сужение расслоения $S \rightarrow \Sigma$ на $h(X) \subset$ $\Sigma$ изоморфно спинорному расслоению $S^{h} \rightarrow X$, сечения которого представляют собой фермионные поля в присутствии тетрадного гравитационного поля $h$. Поэтому сечения расслоения (8) описывают всю совокупность фермионных и гравитационных полей.

Во втором варианте [29], [32] используется следуюший факт. Если $Q$ - ассоциированное с $L X$ групповое расслоение, то сушествует морфизм

$$
\rho: Q \times \Sigma \rightarrow \Sigma,
$$

который сопоставляет всякому тетрадному полю $h$ некоторое фоновое тетрадное поле $h_{0}$ и сечение $q$ расслоения $Q \rightarrow X$, которое отождествляется с гравитационным полем. Дираковские фермионные поля в таком подходе описываются сечениями расслоения $S^{h_{0}} \rightarrow X$, а их динамика в присутствии гравитационного поля $q$ выглядит как движение в эффективном тетрадном поле $\tilde{h}$. 
Мы определим конфигурационное пространство, оператор Дирака и лагранжианы для обоих вариантов калибровочной теории гравитации.

\section{2. ЛОРЕНЦЕВСКАЯ СТРУКТУРА}

Как уже было сказано, теория гравитации строится как калибровочная теория на главном расслоении $L X \rightarrow X$ ориентированных касательных реперов к пространственно-временному многообразию $X$ со структурной группой $G L_{4}$.

Скажем несколько слов об этом расслоении. Всякий его элемент можно представить в виде $H_{a}=H_{a}^{\mu} \partial_{\mu}$, где $\left\{\partial_{\mu}\right\}$ - голономные базисы касательного расслоения $T X$ и $H_{a}^{\mu-}$ матричные элементы представления группы $G L_{4}$ в $R^{4}$. Последние являются координатами на расслоении $L X$ с функциями перехода

$$
H_{a}^{\prime \mu}=\frac{\partial x^{\prime \mu}}{\partial x^{\lambda}} H_{a}^{\lambda}
$$

В этих координатах правое действие структурной группы $G L_{4}$ на $L X$ имеет вид

$$
R_{g}: H_{a}^{\mu} \mapsto H_{b}^{\mu} g_{a}^{b}, \quad g \in G L_{4} .
$$

Реперное расслоение $L X$ наделено канонической $R^{4}$-значной 1-формой

$$
\theta_{L X}=H_{\mu}^{a} d x^{\mu} \otimes t_{a},
$$

где $\left\{t_{a}\right\}$ - фиксированный базис $R^{4}$ и $H_{\mu}^{a}$ - элементы обратной матрицы.

Как и во всякой калибровочной теории, связности на главном реперном расслоении $L X$ являются калибровочными полями в теории гравитации. Они находятся во взаимно однозначном соответствии с линейными связностями $K$ на касательном расслоении $T X$ (или просто на $X$ ). В голономных координатах $\left(x^{\lambda}, \dot{x}^{\lambda}\right)$ на $T X$ эти связности задаются тангенциальнозначными формами

$$
K=d x^{\lambda} \otimes\left(\partial_{\lambda}+K_{\lambda}{ }_{\nu}{ }_{\nu} \dot{x}^{\nu} \dot{\partial}_{\mu}\right)
$$

и представляются сечениями фактор-расслоения

$$
C=J^{1} L X / G L_{4} \rightarrow X
$$

где $J^{1} L X$ - многообразие струй первого порядка сечений расслоения $L X \rightarrow X$ [31]. Расслоение связностей $C$ наделено координатами $\left(x^{\lambda}, k_{\lambda}{ }^{\nu}{ }_{\alpha}\right)$ такими, что координаты $k_{\lambda}{ }^{\nu}{ }_{\alpha} \circ K=K_{\lambda}{ }^{\nu}{ }_{\alpha}$ любого его сечения $K$ представляют собой коэффициенты соответствующей линейной связности (12). Подчеркнем, что расслоение связностей $C$ (13) не ассоциировано с реперным расслоением, но также допускает общие ковариантные преобразования.

Как уже отмечалось, метрическое (тетрадное) поле вводится в калибровочную теорию гравитации путем задания лоренцевской структуры. Лоренцевской структурой на 
пространственно-временном многообразии $X$ называется подрасслоение $L^{h} X$ реперного расслоения $L X$ со структурной группой Лоренца $L$, где $h$ - глобальное сечение фактор-расслоения (5).

Тетрадное поле $h$ определяет атлас $\Psi^{h}=\left\{\left(U_{\zeta}, z_{\zeta}^{h}\right)\right\}$ реперного расслоения $L X$ такой, что локальные сечения $z_{\zeta}^{h}$ расслоения $L X$ принимают значения в лоренцевском подрасслоении $L^{h} X$ и имеют лоренцевские функции перехода. Они называются тетрадными функциями и имеют координатный вид $h_{a}^{\mu}=H^{\mu}{ }_{a} \circ z_{\zeta}^{h}$. Тетрадные функции индуцируют локальную тетрадную форму

$$
z_{\zeta}^{h *} \theta_{L X}=h_{\lambda}^{a} d x^{\lambda} \otimes t_{a}
$$

на $X$, где $\theta_{L X}$ - каноническая форма (11) на $L X$. Эта тетрадная форма, в свою очередь, задает тетрадные кореперы

$$
h^{a}=h_{\mu}^{a}(x) d x^{\mu}, \quad x \in U_{\zeta},
$$

на кокасательном расслоении $T^{*} X$ к $X$. В частности, соотношение (6) принимает вид $g=\eta_{a b} h^{a} \otimes h^{b}$. Таким образом, метрика $g$ сводится к метрике Минковского относительно лоренцевского атласа $\Psi^{h}$ и служит примером лоренцевских инвариантов, упоминаемых в геометрическом принципе эквивалентности.

Рассмотрим теперь калибровочные поля при наличии лоренцевской структуры. Связности на лоренцевском расслоении $L^{h} X$ имеют вид

$$
A_{h}=d x^{\lambda} \otimes\left(\partial_{\lambda}+\frac{1}{2} A_{\lambda}^{a b} \varepsilon_{a b}\right)
$$

где $\varepsilon_{a b}{ }^{c}{ }_{d}=\eta_{a d} \delta_{b}^{c}-\eta_{b d} \delta_{a}^{c}$ - генераторы группы Лоренца. Будем называть их лоренцевскими связностями. С учетом свойства эквивариантности относительно правого действия (10) структурной группы на главное расслоение всякая лоренцевская связность на $L^{h} X$ может быть продолжена до связности на реперном расслоении $L X$, определяя тем самым линейную связность $K(12)$ на $X$ с коэффишиентами

$$
K_{\lambda}{ }^{\mu}{ }_{\nu}=h_{\nu}^{k} \partial_{\lambda} h_{k}^{\mu}+\eta_{k a} h_{b}^{\mu} h_{\nu}^{k} A_{\lambda}^{a b} .
$$

Можно сформулировать и обратное утверждение. Согласно известной теореме [19] линейная связность на $X$ является лоренцевской тогда и только тогда, когда ее группа голономии сводится к группе Лоренца. В то же время можно показать [29], [31], что любая линейная связность $K(12)$ определяет на каждом лоренцевском подрасслоении $L^{h} X$ лоренцевскую связность $A_{h}(14)$ с коэффициентами

$$
A_{\lambda}^{a b}=\frac{1}{2}\left(\eta^{k b} h_{\mu}^{a}-\eta^{k a} h_{\mu}^{b}\right)\left(\partial_{\lambda} h_{k}^{\mu}-h_{k}^{\nu} K_{\lambda}{ }^{\mu}{ }_{\nu}\right) .
$$

Это позволяет использовать в теории гравитации с фермионными полями общие связности, не обусловленные какой-либо спиновой структурой. 


\section{3. УНИВЕРСАЛЬНАЯ СПИНОВАЯ СТРУКТУРА}

Поскольку первая гомотопическая группа группового пространства $G L_{4}$ равна $Z_{2}$, существует универсальная накрываюшая $\widetilde{G L_{4}}$ группы $G L_{4}$ такая, что диаграмма

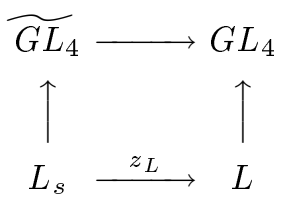

коммутативна. Группа $\widetilde{G L_{4}}$ имеет спинорное представление, элементы которого, называемые "мировыми" спинорами, предлагалось использовать для описания фермионов в теории гравитации [12]. Однако это представление бесконечномерно. Поэтому мы выбрали другой путь.

Рассмотрим двулистное накрытие $\widetilde{L X} \rightarrow X$ реперного расслоения $L X$, которое является главным расслоением со структурной группой $\widetilde{G L_{4}}$ [25], [33], [34]. Поскольку $\Sigma=\widetilde{L X} / L_{\mathrm{S}}$, расслоение

$$
\widetilde{L X} \rightarrow \Sigma
$$

является главным расслоением со структурной группой $L_{s}$. Поэтому имеет место коммутативная диаграмма

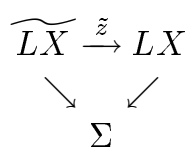

которая задает спиновую структуру на тетрадном расслоении $\Sigma$. Эта спиновая структура единственна и обладает следуюшим свойством [29]-[31]. Для всякого глобального сечения $h$ расслоения $\Sigma \rightarrow X(5)$ сужение главного расслоения $(17)$ на $h(X) \subset \Sigma$ изоморфно главному расслоению $P^{h}(3)$ со структурной группой $L_{s}$. Поэтому спиновая структура (18) называется универсальной.

Рассмотрим ассоциированное с (17) спинорное расслоение $S \rightarrow \Sigma$. Аналогично предыдушему, если $h$ - сечение расслоения $\Sigma \rightarrow X$, то сужение $h^{*} S$ расслоения $S \rightarrow \Sigma$ на $h(X) \subset \Sigma$ является подрасслоением композиционного расслоения $S \rightarrow X$ (8) и изоморфно ассоциированному с $P^{h}$ спинорному расслоению $S^{h}$. Заметим, что $S \rightarrow X$ не является спинорным расслоением. В частности, голономные автоморфизмы реперного расслоения $L X$ однозначно продолжаются на $\widetilde{L X}$ и индуцируют общие ковариантные преобразования расслоения $S \rightarrow X[28]-[31]$.

Построим оператор Дирака на спинорном расслоении $S \rightarrow \Sigma$ так, чтобы его сужение на подрасслоение $S^{h}$ расслоения $S \rightarrow X$ воспроизводило оператор Дирака фермионных полей в присутствии тетрадного поля $h$ и общей линейной связности $K$ на $X$. Напомним, что связности на спинорном расслоении $S^{h} \rightarrow X$ ассоциированы со связностями на главном расслоении $P^{h}$ и находятся во взаимно однозначном соответствии с лоренцевскими связностями на $L^{h} X$. Отсюда следует, что любая лоренцевская связность $A_{h}(14)$ задает спинорную связность

$$
A_{h}=d x^{\lambda} \otimes\left(\partial_{\lambda}+A_{\lambda}^{a b} L_{a b}{ }_{B}{ }_{B} y^{B} \partial_{A}\right), \quad L_{a b}=\frac{1}{4}\left[\gamma_{a}, \gamma_{b}\right],
$$


на расслоении $S^{h}$, наделенном координатами $\left(x^{\lambda}, y^{A}\right)$ относительно лоренцевского атласа $\Psi^{h}=\left\{h_{\lambda}^{a}\right\}$ расслоения $L^{h} X$, продолженного на $P^{h}$. Следовательно, произвольная линейная связность $K(12)$ на $X$ индуцирует лоренцевскую связность на $L^{h} X$ с коэффициентами (16) и соответственно спинорную связность

$$
K_{h}=d x^{\lambda} \otimes\left[\partial_{\lambda}+\frac{1}{4}\left(\eta^{k b} h_{\mu}^{a}-\eta^{k a} h_{\mu}^{b}\right)\left(\partial_{\lambda} h_{k}^{\mu}-h_{k}^{\nu} K_{\lambda}{ }^{\mu}{ }_{\nu}\right) L_{a b}{ }^{A}{ }_{B} y^{B} \partial_{A}\right]
$$

на $S^{h}$ [28], [31], [35]. Спинорная связность (19) определяет на $S^{h}$ ковариантный дифференциал

$$
D: J^{1} S^{h} \rightarrow T^{*} X \otimes S^{h}, \quad D=\left(y_{\lambda}^{A}-A^{a b}{ }_{\lambda} L_{a b}{ }^{A}{ }_{B} y^{B}\right) d x^{\lambda} \otimes \partial_{A},
$$

где $J^{1} S^{h}$ с координатами $\left(x^{\lambda}, y^{A}, y_{\lambda}^{A}\right)$ - многообразие струй сечений расслоения $S^{h} \rightarrow$ $X$. Ковариантный дифференциал $D(21)$ в композиции с представлением (7) задает на расслоении $S^{h}$ оператор Дирака

$$
\begin{gathered}
\Delta_{h}=\gamma_{h} \circ D: J^{1} S^{h} \rightarrow T^{*} X \otimes S^{h} \rightarrow S^{h}, \\
y^{A} \circ \Delta_{h}=h_{a}^{\lambda} \gamma_{B}^{a A}\left(y_{\lambda}^{B}-\frac{1}{2} A_{\lambda}^{a b} L_{a b}{ }_{B}^{A} y^{B}\right)
\end{gathered}
$$

фермионных полей в присутствии фоновых тетрадного поля $h$ и обшей линейной связности $K$.

Обратимся теперь к расслоению $S \rightarrow \Sigma$ и индуцированному из него спинорному расслоению $Y$ над произведением $\Sigma \times C$, где $C$ - расслоение общих линейных связностей (13). Расслоение $Y$ наделено координатами $\left(x^{\lambda}, y^{A}, \sigma_{\mu}^{a}, k_{\lambda}{ }^{\mu}{ }_{\nu}\right)$, где $\left(x^{\lambda}, \sigma_{\mu}^{a}\right)$ - координаты на тетрадном расслоении $\Sigma$ такие, что координаты $\sigma_{\mu}^{a} \circ h$ любого его сечения $h-$ тетрадные функции $h_{\mu}^{a}$. Многообразие струй $J^{1} Y$ расслоения $Y \rightarrow \Sigma \times C \rightarrow X$ является конфигурационным пространством полной системы фермионных, тетрадных и калибровочных полей в калибровочной теории гравитации, где тетрадное поле и соответствующая псевдориманова метрика отождествляются с гравитационным полем. Оператор Дирака на этом конфигурационном пространстве имеет вид [28]-[31]

$$
\begin{gathered}
\Delta_{Y}=\gamma_{\Sigma} \circ \widetilde{D}: J^{1} Y \rightarrow T^{*} X \otimes_{\Sigma} S \rightarrow S \\
y^{B} \circ \Delta_{Y}=\sigma_{a}^{\lambda} \gamma^{a B}{ }_{A}\left[y_{\lambda}^{A}-\frac{1}{4}\left(\eta^{k b} \sigma_{\mu}^{a}-\eta^{k a} \sigma_{\mu}^{b}\right)\left(\sigma_{\lambda k}^{\mu}-\sigma_{k}^{\nu} k_{\lambda}{ }^{\mu}{ }_{\nu}\right) L_{a b}{ }^{A}{ }_{B} y^{B}\right] .
\end{gathered}
$$

Он удовлетворяет предъявленному выше требованию и совпадает с оператором Дирака (22) при ограничении на $h(X) \times K(X) \subset \Sigma \times C$ для фоновых тетрадного поля $h$ и линейной связности $K$.

Лагранжиан калибровочной теории гравитации на конфигурационном пространстве $J^{1} Y$ может быть выбран в виде суммы

$$
L=L_{\mathrm{D}}+L_{\mathrm{AM}}
$$


дираковского лагранжиана

$$
\begin{aligned}
L_{\mathrm{D}}= & \left\{\frac { i } { 2 } \sigma _ { q } ^ { \lambda } \left[y_{A}^{+}\left(\gamma^{0} \gamma^{q}\right)^{A}{ }_{B}\left(y_{\lambda}^{B}-\frac{1}{4}\left(\eta^{k b} \sigma_{\mu}^{a}-\eta^{k a} \sigma_{\mu}^{b}\right)\left(\sigma_{\lambda k}^{\mu}-\sigma_{k}^{\nu} k_{\lambda}{ }^{\mu}{ }_{\nu}\right) L_{a b}{ }^{B}{ }_{C} y^{C}\right)-\right.\right. \\
& \left.-\left(y_{\lambda A}^{+}-\frac{1}{4}\left(\eta^{k b} \sigma_{\mu}^{a}-\eta^{k a} \sigma_{\mu}^{b}\right)\left(\sigma_{\lambda k}^{\mu}-\sigma_{k}^{\nu} k_{\lambda}{ }^{\mu}{ }_{\nu}\right) y_{C}^{+} L_{a b}^{+{ }_{C}{ }_{A}}\right)\left(\gamma^{0} \gamma^{q}\right)^{A}{ }_{B} y^{B}\right]- \\
& \left.-m y_{A}^{+}\left(\gamma^{0}\right)^{A}{ }_{B} y^{B}\right\} \sqrt{|\sigma|}, \quad \sigma=\operatorname{det}\left(\sigma_{\mu \nu}\right)
\end{aligned}
$$

и лагранжиана

$$
L_{\mathrm{AM}}\left(R_{\mu \lambda}{ }^{\alpha}{ }_{\beta}, \sigma^{\mu \nu}\right), \quad \sigma^{\mu \nu}=\sigma_{a}^{\mu} \sigma_{b}^{\nu} \eta^{a b},
$$

аффинно-метрической теории, выражаемого через тензор кривизны линейной связности

$$
R_{\lambda \mu}^{\alpha}{ }_{\beta}=k_{\lambda \mu}{ }^{\alpha}{ }_{\beta}-k_{\mu \lambda}{ }^{\alpha}{ }_{\beta}+k_{\mu}{ }^{\alpha}{ }_{\varepsilon} k_{\lambda}{ }^{\varepsilon}{ }_{\beta}-k_{\lambda}{ }^{\alpha}{ }_{\varepsilon} k_{\mu}{ }^{\varepsilon} \beta
$$

Нетрудно заметить, что

$$
\frac{\partial L_{\mathrm{D}}}{\partial k_{\lambda}{ }_{\nu}}+\frac{\partial L_{\mathrm{D}}}{\partial k_{\nu_{\lambda}}^{\mu}}=0 .
$$

Поэтому дираковский лагранжиан (25) зависит только от тензора кручения $k_{\lambda}{ }^{\mu}{ }_{\nu}-$ $k_{\nu}{ }_{\lambda}{ }_{\lambda}$ линейной связности. Кроме того, полный лагранжиан (24) инвариантен относительно неголономных калибровочных преобразований

$$
k_{\lambda}{ }_{\nu}{ }_{\nu} k_{\lambda}{ }^{\mu}{ }_{\nu}+V_{\lambda} \delta_{\nu}^{\mu}
$$

т.е. обладает так называемой проективной свободой.

\section{4. ГОЛДСТОУНОВСКОЕ ГРАВИТАЦИОННОЕ ПОЛЕ}

Рассмотрим ассоциированное с $L X$ групповое расслоение $Q \rightarrow X$, типичным слоем которого является группа $G L_{4}$, действуюшая на себя по присоединенному представлению. Оно наделено координатами $\left(x^{\lambda}, q^{\lambda}\right)$ как подрасслоение тензорного расслоения $T X \otimes T^{*} X$ и допускает каноническое сечение $q_{0}(x)=\partial_{\mu} \otimes d x^{\mu}$. Задано левое каноническое действие $Q$ на всякое ассоциированное с $L X$ расслоение. В частности, его действие (9) на тетрадное расслоение $\Sigma$ имеет вид

$$
\rho:\left(x^{\lambda}, q_{\mu}^{\lambda}, \sigma_{a}^{\mu}\right) \mapsto\left(x^{\lambda}, q_{\mu}^{\lambda} \sigma_{a}^{\mu}\right) .
$$

Фиксируем теперь тетрадное поле $h$ и обозначим через $\operatorname{Ker}_{h} \rho$ множество тех элементов группового расслоения $Q$, которые оставляют $h$ неподвижным. Это - подрасслоение расслоения $Q$. Обозначим через $\Sigma_{h}$ фактор расслоения $Q$ по $\operatorname{Ker}_{h} \rho$. Это расслоение с координатами $\tilde{\sigma}_{a}^{\mu}=q^{\mu}{ }_{\nu} h_{a}^{\nu}$ изоморфно расслоению $\Sigma$, наделенному лоренцевской структурой ассоциированного с $L^{h} X$ расслоения. В результате может быть определено [29], [32] представление

$$
\begin{aligned}
& \gamma_{Q}:\left(\Sigma_{h} \times T^{*} X\right) \otimes_{\Sigma_{h}}\left(\Sigma_{h} \times S^{h}\right) \rightarrow\left(\Sigma_{h} \times S^{h}\right), \\
& \gamma_{Q}:\left(\tilde{\sigma}, d x^{\mu}\right) \mapsto q^{\mu}{ }_{\nu} h_{a}^{\nu} \gamma^{a}=\tilde{\sigma}_{a}^{\mu} \gamma^{a} .
\end{aligned}
$$


Поэтому сечение $\tilde{h} \neq h$ расслоения $\Sigma_{h}$ можно трактовать как эффективное тетрадное поле, а $\tilde{g}^{\mu \nu}=\tilde{h}_{a}^{\mu} \tilde{h}_{b}^{\nu} \eta_{a b}-$ как эфффективную метрику. При этом $\tilde{h}$ не является новым тетрадным полем, а $\tilde{g}$ - новой метрикой, поскольку ковекторы $\tilde{h}^{a}=\tilde{h}_{\mu}^{a} d x^{\mu}$ реализуются $\gamma$-матрицами в том же представлении, что и ковекторы $h^{a}=h_{\mu}^{a} d x^{\mu}$, а греческие индексы поднимаются и опускаются фоновой метрикой $g^{\mu \nu}=h_{a}^{\mu} h_{b}^{\nu} \eta_{a b}$.

Таким образом, мы приходим к варианту релятивистской теории гравитации (РТГ) $[36],[37]$ в случае фонового тетрадного поля $h$ и динамического гравитационного поля $q$, которое в рамках калибровочной теории можно интерпретировать как голдстоуновское поле. Построим оператор Дирака и полный лагранжиан калибровочной теории с голдстоуновским гравитационным полем.

Рассмотрим спинорное расслоение $S^{h}$, ассоциированное с фоновым тетрадным полем $h$, и спинорную связность $K_{h}(20)$ на $S^{h} \rightarrow X$, индуцированную линейной связностью $K(12)$ на $X$. Используя определяемый этой связностью ковариантный дифференциал $D(21)$ и представление $\gamma_{Q}(27)$, можно построить оператор Дирака

$$
\Delta_{Q}=q_{\mu}^{\lambda} h_{a}^{\mu} \gamma^{a} D_{\lambda}
$$

При ограничении на каноническое сечение $q_{0}(X)$ оператор Дирака (28) сводится к оператору Дирака $\Delta_{h}(22)$ на $S^{h}$ для фермионных полей в присутствии фонового тетрадного поля $h$ и линейной связности $K$.

Таким образом, мы получаем расширение РТГ, где динамическими переменными являются голдстоуновское гравитационное поле $q$, общие линейные связности $K$ и дираковские фермионные поля в присутствии фонового тетрадного поля $h$. Конфигурационным пространством такой модели служит многообразие струй $J^{1} Z$ расслоения

$$
Z=Q \times C \times S^{h}
$$

параметризуемого координатами $\left(x^{\lambda}, q^{\mu}{ }_{\nu}, k_{\lambda}{ }^{\mu}{ }_{\nu}, y^{A}\right)$. Полньй лагранжиан на этом конфигурационном пространстве можно выбрать в виде суммы

$$
L=L_{\mathrm{AM}}+L_{q}(q, g)+L_{\mathrm{D}}
$$

лагранжиана $L_{\mathrm{AM}}$ афффинно-метрической теории, выражаемого через компоненты тензора кривизны (26), сворачиваемые посредством эффективной метрики $\tilde{\sigma}^{\mu \nu}=\tilde{\sigma}_{a}^{\mu} \tilde{\sigma}_{b}^{\nu} \eta^{a b}$, лагранжиана $L_{q}$ голдстоуновского гравитационного поля $q$, в котором свертка осуществляется посредством фоновой метрики $g$, и дираковского лагражиана $L_{\mathrm{D}}(25)$, в котором тетрадное гравитационное поле $\sigma$ заменено на эффективное тетрадное поле $\tilde{\sigma}$. В частности, положив

$$
L_{\mathrm{AM}}=\left(-\lambda_{1} R+\lambda_{2}\right)|\tilde{\sigma}|^{-1 / 2}, \quad L_{q}=\lambda_{3} g_{\mu \nu} \tilde{\sigma}^{\mu \nu}|\sigma|^{-1 / 2}, \quad L_{\mathrm{D}}=0,
$$

где $R=\tilde{\sigma}^{\mu \nu} R_{\mu \alpha \nu}^{\alpha}$, можно воспроизвести обычный лагранжиан РТГ. 


\section{Список литературы}

[1] R. Utiyama. Phys. Rev. 1956. V. 101. P. 1597.

[2] T. Kibble. J. Math. Phys. 1961. V. 2. P. 212.

[3] D. Sciama. Rev. Mod. Phys. 1964. V. 36. P. 463.

[4] F. Hehl, P. von der Heyde, G. Kerlick, J. Nester. Rev. Mod. Phys. 1976. V. 48. P. 394.

[5] E. Lord. Phys. Lett. A. 1978. V. 65. P. 1.

[6] F. Basombrio. Gen. Rel. Grav. 1980. V. 12. P. 109.

[7] L. Norris, R. Fulp, W. Davies. Phys. Lett. A. 1980. V. 79. P. 278.

[8] A. Tseitlin. Phys. Rev. D. 1982. V. 26. P. 3327.

[9] T. Kawai. Gen. Rel. Grav. 1986. V. 18. P. 995; 1987. V. 19. P. 1285.

[10] D. Ivanenko, G. Sardanashvily. Phys. Rep. 1983. V. 94. P. 1.

[11] G. Sardanashvily, O. Zakharov. Gauge Gravitation Theory. Singapore: World Scientific, 1992.

[12] F. Hehl, J. McCrea, E. Mielke, Y. Ne'eman. Phys. Rep. 1995. V. 258. P. 1.

[13] A. Kadić, D. Edelen. A Gauge Theory of Dislocations and Disclinations. Berlin: Springer, 1983.

[14] C. Malyshev. Ann. Phys. 2000. V. 286. P. 249.

[15] G. Sardanashvily, M. Gogberashvily. Mod. Phys. Lett. A. 1987. V. 2. P. 609.

[16] L. Nikolova, V. Rizov. Rep. Math. Phys. 1984. V. 20. P. 287.

[17] M. Keyl. J. Math. Phys. 1991. V. 32. P. 1065.

[18] G. Sardanashvily. J. Math. Phys. 1992. V. 33. P. 1546.

[19] ШІ. Кобаяси, К. Номидзу. Основы дифференциальной геометрии. Т. 1. М.: Наука, 1981.

[20] В. Огиевецкий, И. Полубаринов. ЖЭТФ. 1965. Т. 48. С. 1625.

[21] C. Isham, A. Salam, J. Strathdee. Ann. Phys. 1970. V. 62. P. 98.

[22] A. Trautman. Czech. J. Phys. B. 1979. V. 29. P. 107.

[23] G. Sardanashvily. Phys. Lett. A. 1980. V. 75. P. 257.

[24] S. Avis, C. Isham. Commun. Math. Phys. 1980. V. 72. P. 103.

[25] H. Lawson, M-L. Michelson. Spin Geometry. Princeton: Princeton Univ. Press, 1989.

[26] R. Geroch. J. Math. Phys. 1968. V. 9. P. 1739.

[27] G. Wiston. Int. J. Theor. Phys. 1974. V. 11. P. 341; 1975. V. 12. P. 225.

[28] G. Sardanashvily. Class. Quant. Grav. 1997. V. 14. P. 1371.

[29] G. Sardanashvily. J. Math. Phys. 1998. V. 39. P. 4874.

[30] G. Sardanashvily. Int. J. Theor. Phys. 1998. V. 37. P. 1275.

[31] L. Mangiarotti, G. Sardanashvily. Connections in Classical and Quantum Field Theory. Singapore: World Scientific, 2000.

[32] Г. Сарданашвили. ТМФ. 1998. Т. 114. № 3. С. 470.

[33] L. Dabrowski, R. Percacci. Commun. Math. Phys. 1986. V. 106. P. 691.

[34] S. Switt. J. Math. Phys. 1993. V. 34. P. 3825.

[35] V. Ponomarev, Yu. Obukhov. Gen. Rel. Grav. 1982. V. 14. P. 309.

[36] A. Logunov. Relativistic Theory of Gravity. N.Y.: Nova Science Publ., 1998.

[37] А. Логунов. Теория гравитационного поля. М.: Наука, 2001.

Поступила в редакцию 21.II.2002 г. 